\title{
Inhomogeneous Deformation of Microcrystalline Region in I sotactic Polypropylene Film Revealed by a Simultaneous Kinetic Measurement of Microscopic Infrared Dichroism and Macroscopic Stress
}

\author{
Yihu Song, Yasuyoshi Shigematsu, ${ }^{*}$ Koh-Hei Nitta, ${ }^{* *}$ and Norio Nemoto ${ }^{\dagger}$ \\ Department of Molecular and Material Sciences, IGSES, Kyushu University, Hakozaki, Fukuoka 812-8581, Japan \\ *Production Department III, Nagahama Plant, Mitsubishi Plastic Incorporation, Mitsuya, Nagahama 526-8660, Japan \\ ${ }^{*}$ School of Materials Science, Japan Advanced Institute of Science and Technology, Ishikawa 923-1292, Japan
}

(Received January 15, 2002; Accepted July 8, 2002)

\begin{abstract}
The orientation behaviors of macromolecules in the microcrystalline and the amorphous regions of two isotactic polypropylene films with different degrees of inhomogeneity are studied using an instrument constructed for simultaneous kinetic measurement of microscopic IR (MicIR) dichroism and macroscopic stress at constant elongation rate. Reliability of IR data obtained by the kinetic measurement is demonstrated. The influence of the necking on the molecular orientation is revealed pursuing the time profiles of the MicIR dichroism sampled in a same small sampling area that is initially located at the center of the film but continuously moves during stretching due to the inhomogeneity of the sample. The dramatic molecular orientation in the sampling area is found to mainly occur during the process that the necking passes through this area and is almost independent of the macroscopic stress-strain relationship. The dichroism differences in different locations of the microcrystalline region next to a giant spherulite are shown to exemplify the influence of the inhomogeneity on the molecular orientation behavior.
\end{abstract}

KEY WORDS Isotactic Polypropylene / Microscopic Infrared Dichroism / Orientation / Necking /

Studies of deformation mechanism of polymer materials, in which spatial arrangements of macromolecules are achieved, are of considerable importance for film and fiber processing and related applications. ${ }^{1}$ Recent advances in polymer technologies have made it possible to prepare very thin films without any loss of the desired functions. The microscopic morphology of semicrystalline polymer thin films is inhomogeneous due to the co-existence of the crystalline and the amorphous phases, which is expected to lead to the inhomogeneous deformation.

As far as the inhomogeneous deformation is concerned, the necking must be referred. Uniaxial stretching of polymers toward large strains always gives rise to instability, ${ }^{2}$ appearing as a shear banding at a characteristic angle followed by a stabilized neck formation and propagation. ${ }^{3}$ The necking observed in semi-crystalline polymers is related to various processes such as onset of isolated crystallite slip, cooperation of the slips, crystallite fragmentation and amorphous chain disentanglement as strain is increased. ${ }^{4,5}$ During the stable neck propagation, the lamella slides and then transforms gradually into microfibril. ${ }^{6-8}$ It is also found for poly(butylene terephthalate) (PBT) that the spherulitic structure is only partially destructed in the necked region. ${ }^{9}$

The influence of necking on the molecular orientation was noticed at the earlier stage of the rheo-optical studies using the traditional Fourier-transform infrared (FT-IR) technology. ${ }^{10-12}$ Owing to the limitation of the traditional FT-IR method with a sampling area on the centimeter level, however, it was impossible to discuss quantitatively the molecular orientation process in semi-crystalline polymers as neck propagates.

In our laboratory, a new equipment for simultaneous in-situ kinetic measurement of the microscopic infrared (MicIR) dichroism and the macroscopic stress of polymer films subjected to uniaxial elongation at constant rate has been constructed recently, as is described in an earlier paper in detail. ${ }^{13}$ Use of the microscope allows to pursue MicIR spectrum from a desired sampling area with a size of several hundreds or even several decades of micrometer order. We are able to investigate the molecular orientations within this small area and also to detect the deformation of this small area continuously while the film is undergoing the inhomogeneous deformation. A series of works are planned to demonstrate that this equipment is a very powerful instrument for studying of the deformation mechanism of semi-crystalline polymers as well as polymer alloys with various chemical and component compositions and also with various morphologies. In this paper, we first demonstrate the reliability of this instrument using isotactic polypropylene (iPP) as a model polymer in consideration of the well documented vibrational assignments and the elongational infrared dichroism in

${ }^{\dagger}$ To whom correspondence should be addressed (E-mail: nemo@mm.kyushu-u.ac.jp). 
literature. ${ }^{10-12,14-17}$ We then report on the molecular orientation of inhomogeneous iPP films annealed at two conditions with special attention to a relationship between the molecular orientation and the necking.

\section{EXPERIMENTAL}

\section{Materials}

Isotactic polypropylene (iPP) with an average molecular weight $M_{\mathrm{w}}=3.7 \times 10^{5}$ was used. The iPP pellets were thermally compressed at $210^{\circ} \mathrm{C}$ for $5 \mathrm{~min}$ to form the films with an average thickness of about $30 \mu \mathrm{m}$, which were then annealed at two different conditions. When annealed at $135^{\circ} \mathrm{C}$ for $30 \mathrm{~min}$, the film (coded film-A) obtained was apparently homogeneous and only contained microcrystals of a size less than $10 \mu \mathrm{m}$ in diameter. When annealed at $145^{\circ} \mathrm{C}$ for $240 \mathrm{~min}$, the film (coded film-B) was found to contain giant spherulites with the $\alpha$ type of crystalline structure and a diameter of about $300 \mu \mathrm{m}$. Figures 1 and 2 show the typical polarized optical micrographs of the original (a) and the stretched (b) specimens for the film-A and the film-B, respectively. The degree of inhomogeneity in the film-A is about $10 \mu \mathrm{m}$, which is much larger than a contour length of the iPP molecule. On the other hand, the film-B is inhomogeneous to the extent as is detectable by direct microscope observation.

\section{Apparatus and Method}

The setup details of the equipment were described in an earlier paper. ${ }^{13}$ In brief, the IR light beam provided by the FT-IR spectrometer (FTS 6000, Bio-Rad) was passed through a polarizer and a photo-elastic modulator (PEM-90 ZnSe, Hinds Instru.) and irradiated vertically on the film from the bottom. The transmitted light from the center spot of the film was detected using a mercury-cadmium-telluride (MCT) detector installed on an infrared microscope (UMA 500, Bio-rad). By setting the peak retardation of the modulator as the halfwave, the transmitted light was divided into a broad band signal of $A C(t)=A_{\|}(t)-A_{\perp}(t)$ and a low pass signal of $D C(t)=A_{\|}(t)+A_{\perp}(t)$, where $t$ denotes the elongation time, and $A_{\|}$and $A_{\perp}$ are the polarized spectra with the IR radiation polarized parallel and perpendicular to the draw direction, respectively. After passing through a lock-in amplifier (LIA SR510, Stanford Co.) and a ratio circuit respectively, the $A C(t)$ and $D C(t)$ outputs were changed to analog voltages and alternatively sampled in a computer with a sampling interval of about 30 seconds. This is the latest modification of the earlier equipment. $^{13}$

We define the sequential and alternative sampling of the $A C(t)$ and $D C(t)$ spectra during elongation, as de- scribed above, as the kinetic scan. The intensities of the specified absorption bands given by the kinetic scan mode were relative values because of the signal amplification by a series of electric devices. We measured the $A_{\|}$and $A_{\perp}$ spectra of the specimen in the quiescent state after the elongation was stopped and used them to correct the relative values to the absolute ones.

The FT-IR method offers a very efficient technique for measuring the structural or the polarized spectra rapidly called the rapid scan, which needs the acquisition time as $0.3 \mathrm{~s}$ for single scan under $4 \mathrm{~cm}^{-1}$ resolution. In order to examine the reliability of the results obtained by the kinetic scan, we also performed the rapid scan to measure the $A_{\|}(t)$ and $A_{\perp}(t)$ spectra during elongation at an interval of one minute rotating the polarizer to $0^{\circ}$ and $90^{\circ}$ alternatively. In this measurement, the PEM controller was closed and the predetermined sampling area was always adjusted to the viewing field before starting a rapid scan. This type of measurement is tedious and reliable only if the elongation rate is small enough, which is the case in this work.

The resolution of the infrared spectrometer was set $4 \mathrm{~cm}^{-1}$. The viewing field for sampling of the MicIR dichroism signals was set as $200 \times 200 \mu \mathrm{m}^{2}$ and $100 \times 100 \mu \mathrm{m}^{2}$ for the film-A and the film-B, respectively. During the kinetic scan of the $A C(t)$ and $D C(t)$ spectra, the scan-co-addition number was set 10 , which gave a considerable restriction to the time resolution but was found necessary for collection of the accurate data. For the rapid scan of the $A_{\|}(t)$ and $A_{\perp}(t)$ spectra in the quiescent state and during elongation, the scanco-addition number was set 16 and 8, respectively.

The films were carefully cut to the rectangularshaped specimens with a size of $10 \times 3 \mathrm{~mm}^{2}$ (length $\times$ width), which were fastened between the two clamps of the mechanical device with a sample cell in the same compartment of the FT-IR system and were uniaxially stretched with a constant strain rate of $0.066 \mathrm{~min}^{-1}$ at $30.0 \pm 0.1^{\circ} \mathrm{C}$. Only one isolated giant spherulite with an area content of $5 \%$ was cut into the elongation region of the film-B between the two clamps in order to avoid the boundary damage occurred at the earlier stage of elongation and to stretch the specimens to a macroscopic strain as the same level as the film-A. The two clamps of the mechanical device moved symmetrically from the specimen center. IR light intensity transmitted from the center of the film was exclusively measured, if there is no specific statement about the sampling area. This comes form consideration that the specimen center does not shift for homogeneous deformation. During elongation, we could observe the specimen through the IR microscope by switching the view/detection selection optics. 


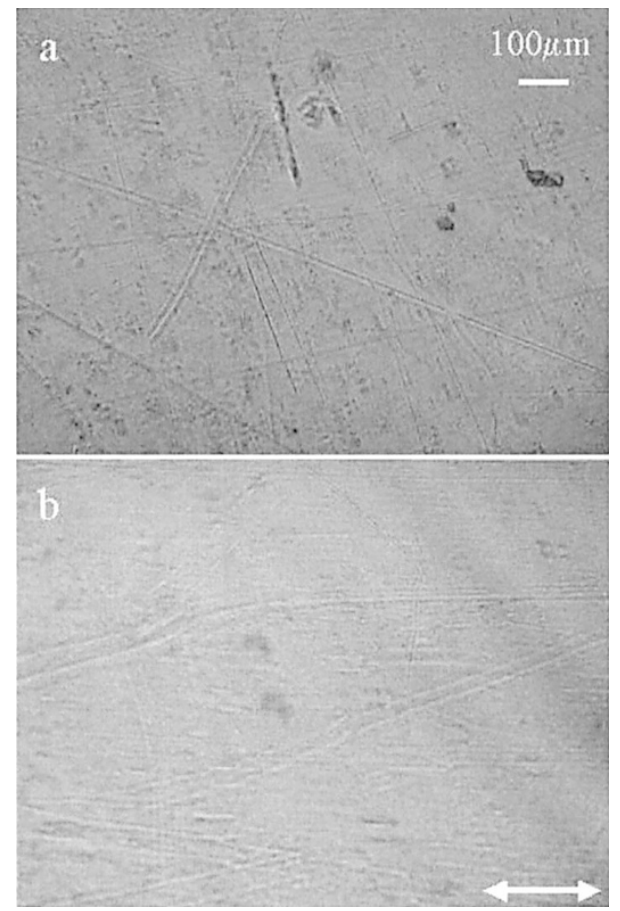

Figure 1. Polarized optical micrographs of the original film-A (a) and of the film stretched to macroscopic strain $\varepsilon=12$ (b). The heavy arrow shows the elongation direction.

Such design was found to be very convenient for a study of inhomogeneous deformation in which the pre-determined sampling area was continuously shifted as the film was stretched. A desired sampling area was always adjusted to the viewing field at a given time interval moving the $x-y-z$ stage where the sample cell was mounted. This adjustment allowed us to obtain the IR data from the same sampling area as accurately as possible.

As seen from Figures 1 and 2, some of the crystal aggregates with a size of several decades of micron could not be destructured completely after the film was stretched to the macroscopic strain $\varepsilon=12$. One of the crystal aggregates was chosen as a reference of the sampling area.

The degrees of the crystallinity of the films were determined from the heat of fusion obtained from analysis of a DSC (DSC8240-TAS100, Rigaku Co.) curve measured at a heating rate of $5^{\circ} \mathrm{C} \mathrm{min}^{-1}$.

Morphologies of both the film-A and the film-B before and after uniaxial stretching were observed using a polarizing optical microscope (Nikon, Poh22326) with a sensitive colour plate. The color appearing on the micrographs of the stretched specimens, especially of the microcrystalline region, may change due to a difference in angle between the stretching axis and the direction of the polarizer.

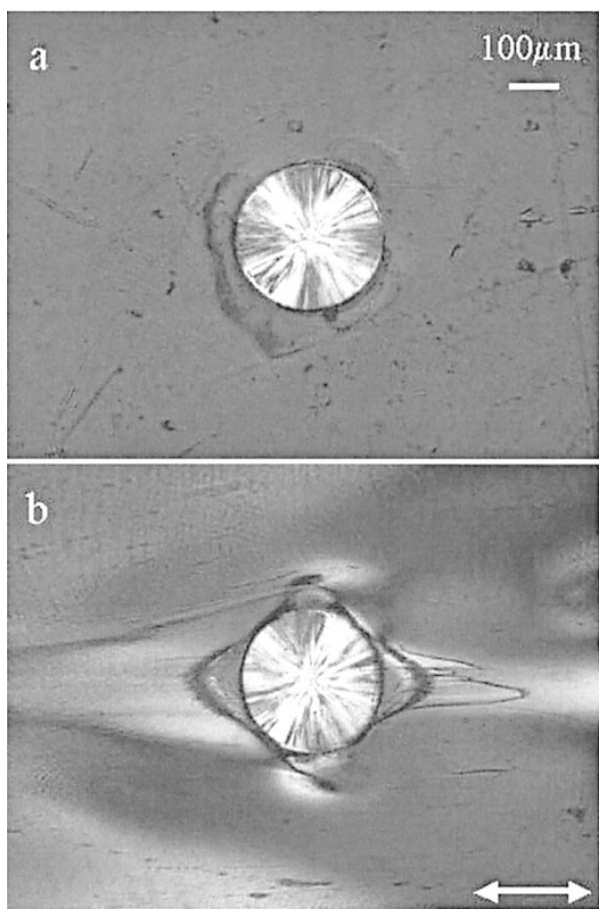

Figure 2. Polarized optical micrographs of the original film-B (a) and of the film stretched to macroscopic strain $\varepsilon=12$ (b). The heavy arrow shows the elongation direction. Only the morphology around the isolated giant spherulite is shown.

\section{RESULTS AND DISCUSSION}

\section{Reliability of the Kinetic Scan Mode}

Time profiles of the MicIR dichroism were evaluated in terms of peak height intensities of the $A C(t)$ and $D C(t)$ in the kinetic scan, or the $A_{\|}(t)$ and $A_{\perp}(t)$ in the rapid scan. We define a dichroic function as ${ }^{13}$

$$
A(t)=\frac{A C(t)}{D C(t)}
$$

which is related to the dichroic ratio $R(t)=A_{\|}(t) / A_{\perp}(t)$ by

$$
A(t)=\frac{R(t)-1}{R(t)+1}
$$

Examples of the $D C(t)$ and $A(t)$ spectra are shown in Figure 3.

From the kinetic scan, the Hermans orientation function, $f(t),{ }^{14}$ for a particular absorption band can be derived as

$$
f(t)=\frac{2 A(t)}{3-A(t)} \frac{R_{0}+2}{R_{0}-1}
$$

where $R_{0}=2 \cot ^{2} \psi$ is the perfect dichroic ratio of a transition moment making an angle $\psi$ with the direction of the molecular main chain axis. Based on eq 3 along with another expression of the Hermans orientation function, ${ }^{14}$

$$
f(t)=\frac{3 \overline{\cos ^{2} \theta}-1}{2}
$$



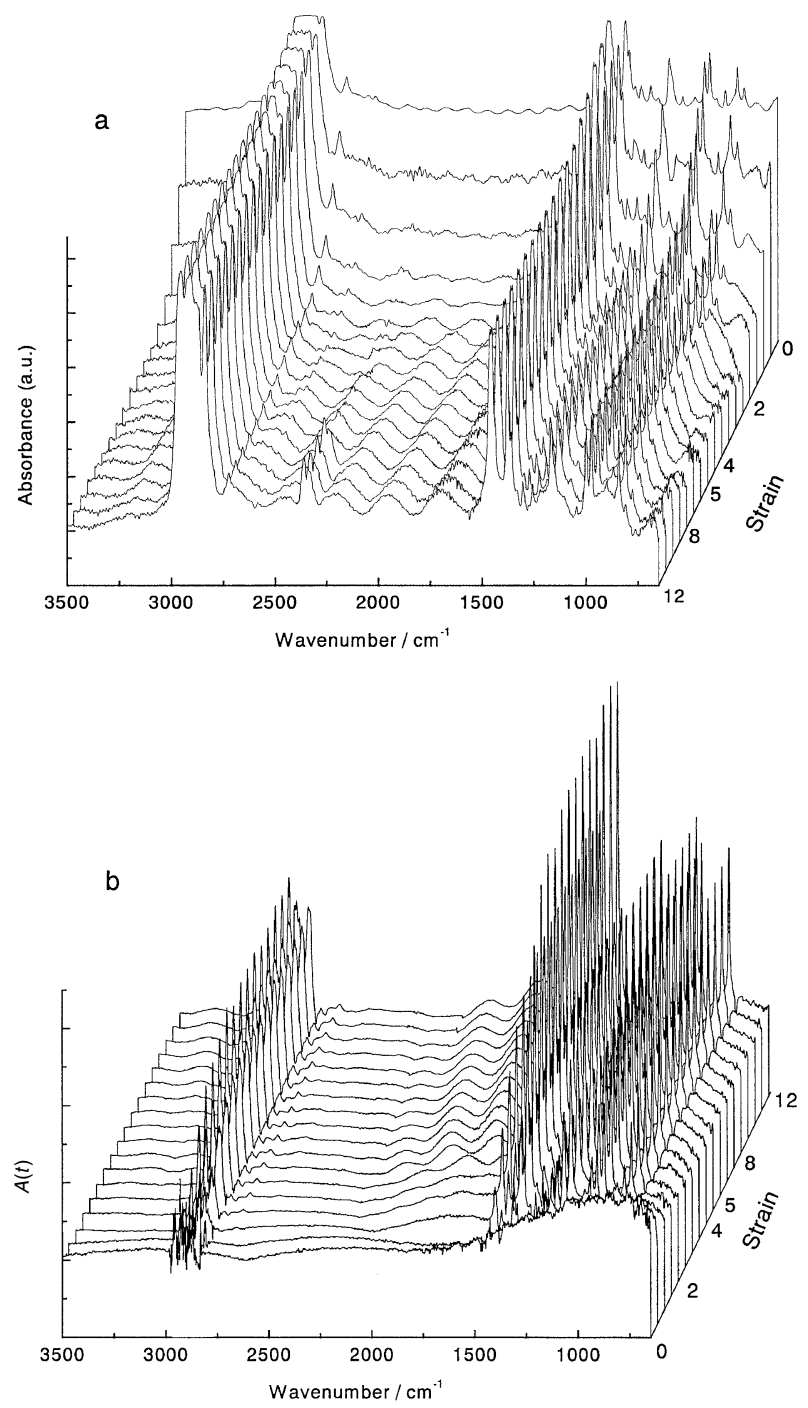

Figure 3. $D C(t)$ (a) and $A(t)(\mathrm{b})$ spectra of the iPP film-A at various strains $\varepsilon$.

we can determine the orientation angles $\theta$ in the particular phases, i.e. the crystalline and the amorphous phases and also in both the crystalline and the amorphous phases hereafter denoted as the averaged phase.

The nominal stress-strain $(\sigma-\varepsilon)$ curves of two specimens of the film-A are given in Figure 4. The two curves collapse to each other when strain $\varepsilon<0.15$, suggesting that the films are uniformly deformed in the linear-elastic deformation region. The stress peaks at $\varepsilon=0.15$ reveal the occurrence of the yielding, followed by a stress softening up to $\varepsilon=0.75$. Although the two specimens are cut out from the same piece of the large original film, the stress starts to give slightly different strain dependences even before the yielding point is reached.

The reliability of the kinetic scan, which is our unique working mode, can be evaluated by comparing the results from the kinetic scan with those from the rapid scan, under the situation that the sampling areas in the two films undergo the similar local de-

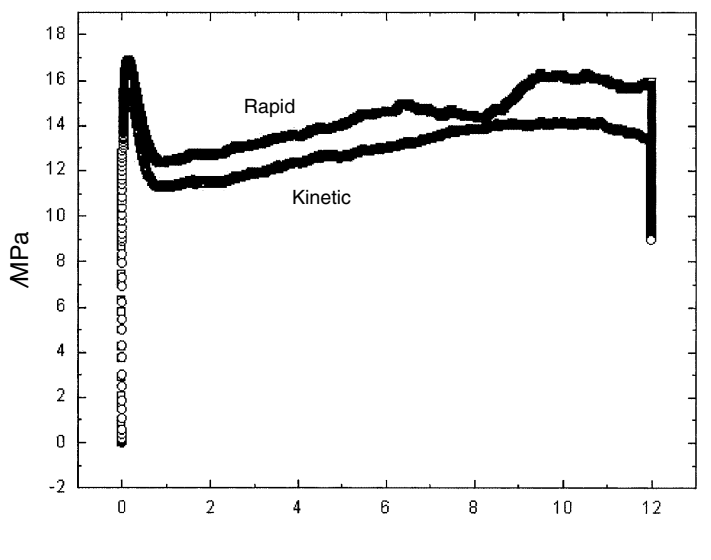

Figure 4. Nominal stress-strain, $\sigma-\varepsilon$, curves of the film-A simultaneously obtained during the IR dichroism measurements by the rapid and the kinetic scan modes.
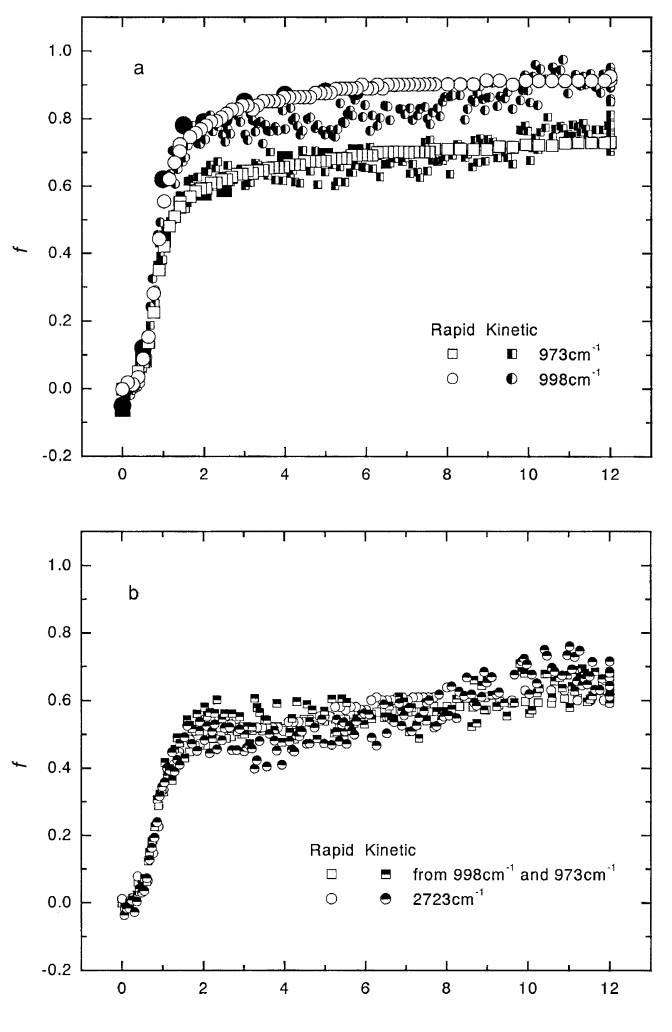

Figure 5. Hermans orientation functions $f(t)$ in the averaged and the crystalline phases (a) and in the amorphous phase (b) as a function of strain $\varepsilon$ for the film-A. Open symbols $(\square, \bigcirc)$ represent the data form the rapid scan mode and half-filled symbols $(\mathbf{\square}, \mathbf{O})$ the data from the kinetic scan mode. The solid symbols $(\mathbf{\square}, \mathbf{0})$ are the data from refs 20 and 22 .

formations during stretching of the specimens. The two specimens in Figure 4 satisfy such a condition, in which the initial necking takes place in the center of the film where the sampling area is located. In Figure 5a, the Hermans orientation functions $f(t)$ from the two $\pi$ bands at $973 \mathrm{~cm}^{-1}$ and $998 \mathrm{~cm}^{-1}$, which are assigned to the vibration modes in the averaged and the crystalline phases respectively, ${ }^{14-16}$ are plotted against strain for 
the both scan modes. The orientation function from the $973 \mathrm{~cm}^{-1}$ band measured by the two scan modes almost follows the same profile, whereas a small difference in the regime from $\varepsilon=2.5$ to $\varepsilon=9.5$ is observed for the orientation function from the $998 \mathrm{~cm}^{-1}$ band. This difference might be related to the stress-strain curves in the same strain region in Figure 4. The orientation behavior in the amorphous phase can be either characterized using the dichroism profile of the $2723 \mathrm{~cm}^{-1}$ $\sigma$-band with $\psi=90^{\circ},{ }^{15}$ or estimated from the orientation functions of the $973 \mathrm{~cm}^{-1}$ and $998 \mathrm{~cm}^{-1}$ bands through $^{13,15,16}$

$$
f_{\mathrm{av}}(t)=V_{\mathrm{c}} f_{\mathrm{c}}(t)+\left(1-V_{\mathrm{c}}\right) f_{\mathrm{am}}(t)
$$

where $V_{\mathrm{c}}$ is the volume fraction of the crystalline phase and was determined as 0.39 for the film-A, and 0.41 for the film-B from the DSC measurement before elongation, and $f_{\mathrm{av}}(t), f_{\mathrm{c}}(t)$, and $f_{\mathrm{am}}(t)$ are the Hermans orientation functions in the averaged, the crystalline and the amorphous phases, respectively. The orientation function of the amorphous phase is presented in Figure 5b, showing that the two scan modes give the same profile. The results in Figure 5 may confirm that we have measured the $A C(t)$ and $D C(t)$ spectra correctly. Despite the slightly scattered characteristic due to on/off switching of the selection optics for observation and adjustment of the sampling area, the dichroism data from the kinetic scan are reliable almost to the same extent as those from the rapid scan.

\section{Effect of Necking on MicIR Dichroism}

In Figure 6, the orientation angles $\theta$ and the stress $\sigma$ simultaneously measured for a specimen of the filmA are plotted against strain $\varepsilon$. At a first glance, the changes in orientation angle appear very exceptional because $\theta_{\mathrm{c}}$ and $\theta_{\mathrm{am}}$, the orientation angles in the

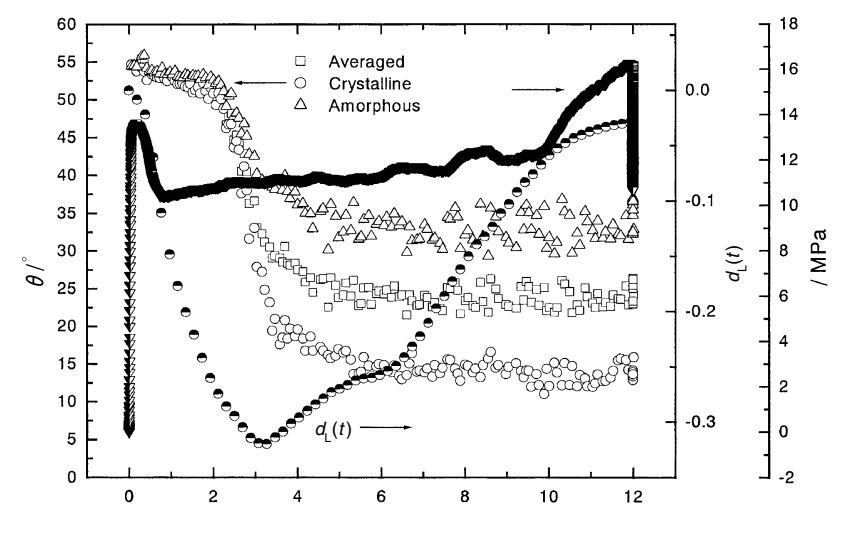

Figure 6. Orientation angles $\theta$ in the averaged, the crystalline and the amorphous phases and stress $\sigma$ as a function of strain $\varepsilon$ for the film-A. The relative displacement of the sampling area $d_{\mathrm{L}}(t)$ is also plotted against strain $\varepsilon$. crystalline and the amorphous phases respectively, decreases only by $5^{\circ}$ and $3^{\circ}$ up to $\varepsilon=2$, whereas the $\sigma-\varepsilon$ behavior already entered the stress-plateau region from $\varepsilon=0.8$ after the stress yielding at $\varepsilon=0.14$. However, it was a rare case that a drastic decrease in $\theta$ started just after $\sigma$ exhibited yielding. Many repeated measurements disclosed that molecular orientation in the original center of the film might take place at various strains despite the similar $\sigma-\varepsilon$ behavior. This peculiar orientation behavior can be interpreted from consideration about the appearance and propagation of a neck.

Corresponding to inhomogeneous deformation of the iPP film, the sampling area was found to never stay under the viewing field once the necking occurred. As a measure of inhomogeneous deformation during uniaxial stretching, we define a parameter, $d_{\mathrm{L}}(t)$, named the relative displacement of the sampling area,

$$
d_{\mathrm{L}}(t)=\frac{D_{\mathrm{L}}(t)}{L(t)}=\frac{D_{\mathrm{L}}(t)}{(\varepsilon+1) L_{0}}
$$

where $D_{\mathrm{L}}(t)$ is the displacement of the specimen center along the stretching axis relative to the sampling area that is fixed in the laboratory coordinates during the entire kinetic measurement, and $L(t)$ and $L_{0}$ are the film lengths at time $t$ and zero, respectively. The $d_{\mathrm{L}}(t)$ remains zero so long as the film undergoes homogeneous deformation. When the sampling area is set at the center of the film taken as the origin of the coordinate along the stretching axis and the necking takes place in the left (right) hand side of the film, $d_{\mathrm{L}}(t)$ takes a positive (negative) value and its absolute magnitude increases as the neck propagates. The time profile of $d_{\mathrm{L}}(t)$ is closely related to the propagation rate of the neck and its passage through the sampling area as well as the macroscopic strain rate. Mathematical relationship shall be discussed in a separate paper in detail.

The parameter $d_{\mathrm{L}}(t)$ plotted against strain in Figure 6 shows that the sampling area departs from the specimen center and moves toward one direction along the stretching axis until $\varepsilon=3.2$. Comparison of the MicIR dichroism data with the $d_{\mathrm{L}}(t)$ profile suggests that the macromolecular chains and the crystallites being present in the end of the sampling area are forced to enter the neck shoulder at $\varepsilon=2.1$. After the neck passes by at $\varepsilon=3.2$, the orientation angles decrease slowly and approach to values of a highly oriented state in a wide strain range where the neck propagates continuously so as to deform the entire film. The significant strain-hardening appearing after $\varepsilon=10$ is not followed by a further molecular orientation, which is different from the previous results ${ }^{10-12}$ and suggests that the further molecular orientation in the full length scale of the specimen is not necessary for the strain-hardening. 


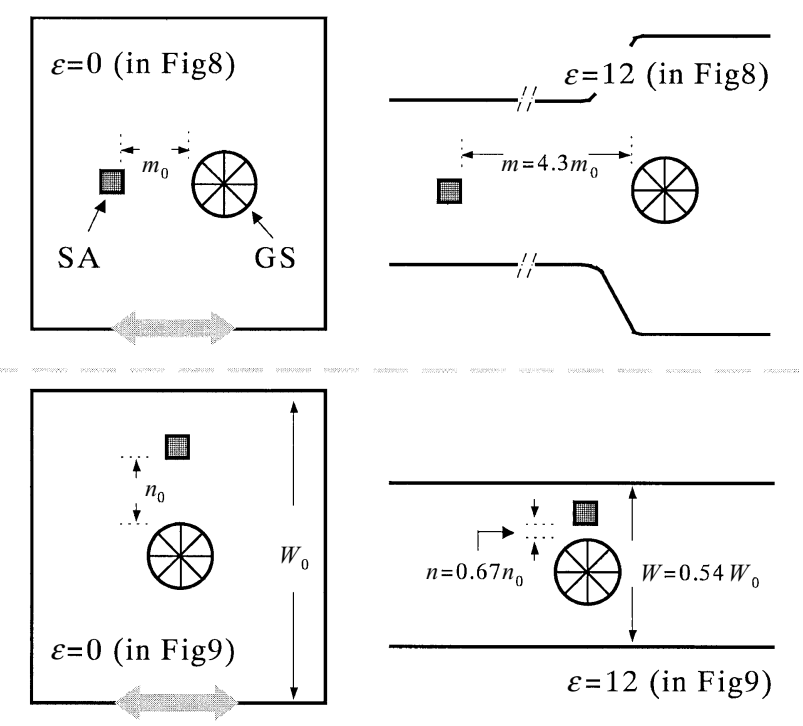

Figure 7. A schematic graph showing the sampling area (SA) in the vicinity of the giant spherulite (GS) in Figures 8 and 9 for the speciments at strains 0 and 12 . The heavy arrow shows the elongation direction and the local deformations between SA and GS observed by the microscope are given. The parameter $m$ and $n$ denote the distance between SA and GS, and $W$ denotes the film width. The subscript zero indicates the parameters before elongation with $m_{0}=n_{0}=280 \mu \mathrm{m}$.

Due to the presence of small spherulites with a size of several microns ${ }^{18,19}$ in the film-A, the local mechanical strength varies from position to position. The necking may be formed at a position with the weakest local strength. In the film-B containing one isolated giant spherulite in its center, the local strength in the vicinity of the spherulite is higher than that in the microcrystalline region so that the initial necking rarely occurs at the position where the spherulite is located.

The necking leads to an inhomogeneous morphology distribution along the stretching axis and also an inhomogeneous morphology evolution of a local area under observation. The morphology of the film-A at large strains may become apparently homogeneous again after the necking extends to the entire specimen, as shown in Figure 1b. Contrarily, the morphology of the microcrystalline region around the giant spherulite becomes always inhomogeneous due to uniaxial stretching, as shown in Figure $2 b$. The microcrystalline region closely next to the spherulite equator is less deformed than that next to the spherulite polar. It is obvious that this kind of inhomogeneous deformation comes from a special stress distribution during the necking passage through the giant spherulite. It is therefore meaningful to investigate the molecular orientation in the microcrystalline region in the vicinity of the giant spherulite. Using two different arrangements of the sampling area and the giant spherulite as schematically presented in Figure 7, the orientation angles, together with the stress

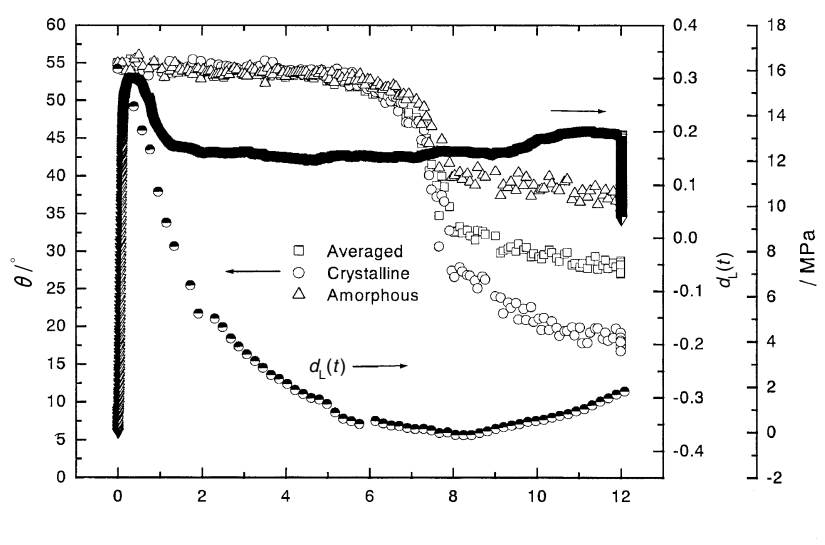

Figure 8. Strain $\varepsilon$ dependence of orientation angles $\theta$ in the averaged, the crystalline and the amorphous phases as well as stress $\sigma$ and $d_{\mathrm{L}}(t)$ in the microcrystalline region of the film-B containing an $\alpha$-spherulite of $5 \%$ in area. The local arrangements of the sampling area and the giant spherulite are schematically shown in Figure 7.

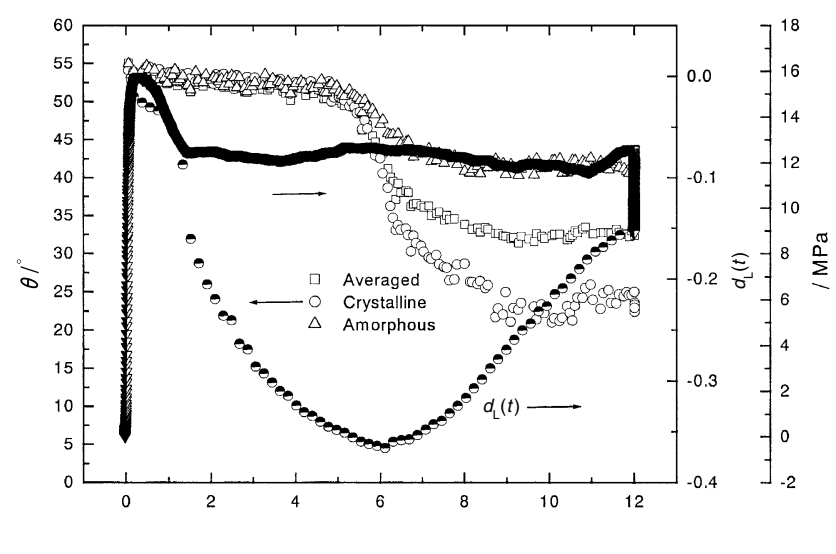

Figure 9. Strain $\varepsilon$ dependence of orientation angles $\theta$ in the averaged, the crystalline and the amorphous phases as well as stress $\sigma$ and $d_{\mathrm{L}}(t)$ in the microcrystalline region of the film-B containing an $\alpha$-spherulite of $5 \%$ in area. The local arrangements of the sampling area and the giant spherulite are schematically shown in Figure 7.

and the $d_{\mathrm{L}}(t)$, as a function of strain, are shown in Figures 8 and 9, respectively. Similar to the result in Figure 6 , the dramatic decreases in orientation angles are mainly associated with the propagation of the necking through the sampling area as revealed by the inversion of the $d_{\mathrm{L}}(t)$ parameter, irrespective of the macroscopic strain. The necking passage induces a molecular orientation with a higher extent in the microcrystalline region next to the spherulite polar than that next to the spherulite equator. After movement into the necked region, the amorphous phase in the microcrystalline region next to the spherulite polar can be slightly deformed, followed by a marked lamellar rotation toward the stretching axis. However, the further deformation of the amorphous phase in the microcrystalline region next to the spherulite equator is not as distinct as that next to the spherulite polar and the lamellar orientation seems to level off at large strains. 


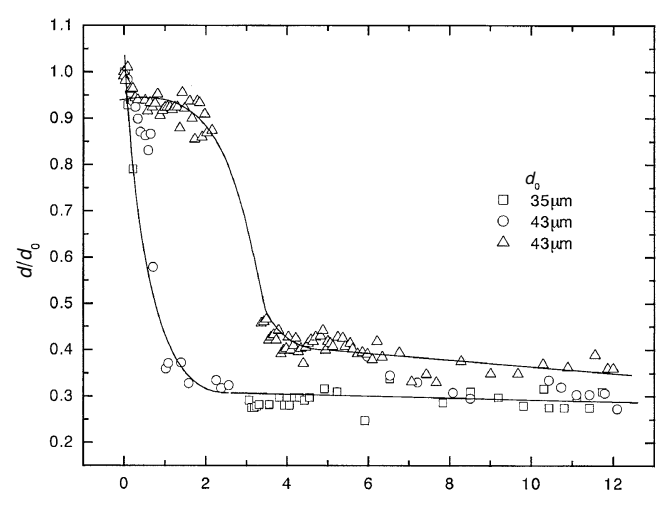

Figure 10. Strain $\varepsilon$ dependence of relative film thickness $d / d_{0}$ obtained from three specimens of the film-A with various initial thicknesses $d_{0}$.

\section{Effect of Necking on Sample Geometry}

The inhomogeneities of both morphology and deformation in semi-crystalline polymers have been known for a long time. ${ }^{20-23}$ From the interference pattern appearing in the $D C(t)$ spectra (as shown in Figure 3a), we can determine the continuous change of the film thickness in the sampling area during the kinetic scan. ${ }^{13}$ The interference pattern usually does not appear in the strain region where the necking is passing through the sampling area due to the thickness gradient which interferes the repeated reflection of the IR beam between the two surfaces. Figure 10 shows the strain dependence of the film thickness obtained from three specimens of the film-A. The striking decrease in thickness occurs only when the sampling area is involved in the necking. When the initial necking occurs far away from the sampling area, the thickness in the sampling area slowly decreases and the deformation is rather close to the elastic one, quite different from that in the neck. Almost the same trend has been found for the film-B during necking.

Figure 11 shows the changes in film width at a predetermined position during elongation, together with the respective $\sigma-\varepsilon$ curves. For the specimen of the filmA, the measuring point was carefully selected at the position where the initial necking took place. The specimen of the film-B contained one giant spherulite, thus, for comparison with the results from the film-A, the spherulite was deliberately placed outside the stretching region between the two clamps. The initial necking appeared at a position far away from the specimen center and another neck appeared from about $\varepsilon=4$. Regardless of the remarkable differences in $\sigma-\varepsilon$ behaviors in Figure 11, the width decreases linearly in the linear-elastic deformation region. From the plastic deformation region, two different tendencies are observed for the neck and the outer section that are not suffered from necking yet. The width in the outer section de-

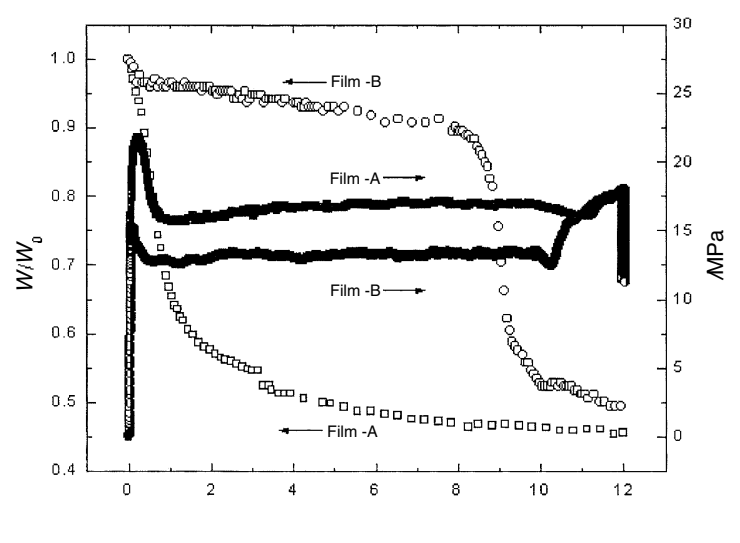

Figure 11. Strain $\varepsilon$ dependence of relative film width $W / W_{0}$ and stress $\sigma$ for the film-A and the film-B.

creases still linearly but slowly, whereas the width profile in the necked region transforms to the exponential decay law from $\varepsilon=1$. The relative widths of the neck at $\varepsilon=12$ are 0.46 and 0.5 for the film-A and the film$\mathrm{B}$, respectively, which are comparable to the result of 0.45 at true strain of 4 obtained by Gaucher-Miri in polyethylene using a different method. ${ }^{26}$ The relative thickness in Figure 10 decreases down to a value in the range from 0.30 to 0.38 , considerably smaller than the relative width. Here a fundamental but more scientific problem remains as why the necking leads to an asymmetrical shrinkage in cross-section while propagating along the elongation axis.

\section{Effect of Morphology on Molecular Orientation}

The considerably slow decreases of the film thickness and width in the neck and also the outer section suggest that these two sections are geometrically stable to some extent compared with the neck shoulder where the significant geometrical evolution is seen. It is reasonable to imagine that molecular orientations are induced by the local strain in the sampling area during a time interval that the area passes from the outer section to the neck via the neck shoulder. However, it is difficult to estimate the local longitudinal strain strictly due to the complicated geometrical evolution of a local area under observation against the macroscopic strain, as revealed in Figures 10 and 11. We here attempt to evaluate the influence of the local strain in the sampling area using the relative thickness, which can be conveniently obtained from the structural absorbances, $A_{0}(t)$, of the properly selected absorbance bands. We find that the structure transformation is negligible for uniaxially stretching of iPP films and there are no essential differences in the normalized structural absorbance, $A_{0}(t) / A_{0}(0)$, of the three bands at $973 \mathrm{~cm}^{-1}, 998 \mathrm{~cm}^{-1}$, and $2723 \mathrm{~cm}^{-1}$. Considering $A_{0}(t) / A_{0}(0)$ of the $973 \mathrm{~cm}^{-1}$ band as representing a 


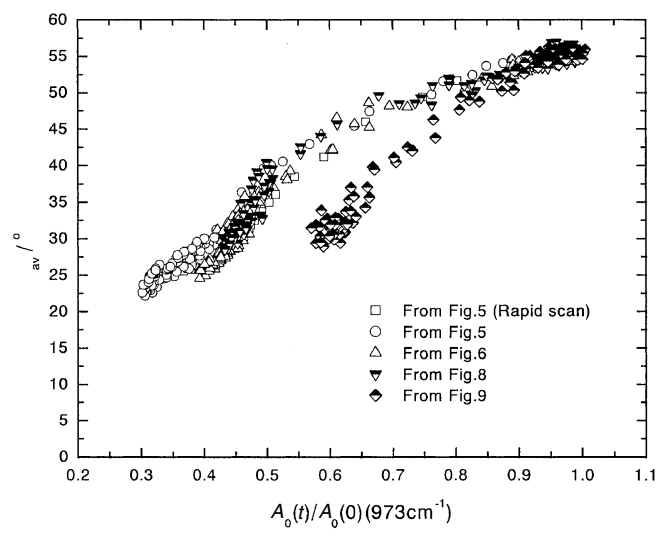

Figure 12. Relationship between the orientation angle in the averaged phase $\theta_{\mathrm{av}}$ and the normalized structural absorbance $A_{0}(t) / A_{0}(0)$ of the band at $973 \mathrm{~cm}^{-1}$.

change in thickness and $\theta_{\mathrm{av}}$, the orientation angle of the averaged phase, as a reference of the average molecular orientation in the sampling area, we replot the results in Figures 5, 6, 8, and 9 in Figure 12. Similar results have been found for $\theta_{\mathrm{c}}$ and $\theta_{\mathrm{am}}$. As shown in Figure 12, the orientation angles are overlapped onto one composite curve for the data given in Figures 5, 6, and 8 during the process of the necking passage through the sampling area even though data scattering is not small, but not for the data given in Figure 9. The former results demonstrate that the molecular orientations at the neck shoulder are controlled by the same mechanisms. On the other hand, the exception for Figure 9 may suggest that the molecular orientations in the microcrystalline region surrounding a giant spherulite are strongly dependent on the location where the MicIR dichroism is sampled. The giant spherulite prevents the microcrystalline region next to the spherulite equator from the shrinkage in thickness (as shown in Figure 2b), therefore the molecules are less deformed than the microcrystalline region next to the spherulite polar and also than the film-A. After the sampling area enters the neck (such as $d / d_{0}<0.45$ ), the data may become scattered due to various orientation mechanisms appearing in the neck entity. It is shown that, even though the necking is considerably crucial for the molecular orientation in iPP films, morphology also plays an important role in the molecular deformation in a local area during and after the necking passage.

The spatial variation of the deformation along the elongation axis is strongly related to the original location of a local area under observation with respect to the position of the initial necking. ${ }^{26}$ Even though the necking occurs at different morphological scales, ${ }^{10,27,28}$ it is associated tightly with the molecular orientation that stipulates the local deformations along the neck. The rapid molecular orientation is induced by the prominent local deformation of the amorphous phase and the rotation of the crystallites that are the concomitants of neck propagation. The results make it clear that the macroscopic strain softening and hardening have no essential relationships with the microscopic molecular orientations. The inhomogeneity of the iPP film is shown by the in-situ kinetic measurement to have a crucial influence on the molecular orientation behavior.

Acknowledgment. This work has been partially supported by the Grant-in-Aid for the Scientific Research from Ministry of Education, Culture, Sports, Science and Technology (No. 10305070) and one of the authors, Y. Song, is grateful to JSPS for the Grant-inAid for JSPS Fellows relating to JSPS Fellowship for Foreign Researchers (No. 12000317).

\section{REFERENCES}

1. L. A. Utracki, "Polymer Alloys and Blends", Hanser, Gardner Publications, Munich, 1989.

2. J. Lu and K. Ravi-Chandar, Int. J. Solids Struc., 36, 391 (1999).

3. Y. Tomita, Int. J. Mechanical Sci., 42, 1455 (2000).

4. R. Hiss, S. Hobeika, C. Lynn, and G. Strobl, Macromolecules, 32, 4390 (1999).

5. R. H. Oliey and D. C. Bassett, J. Macromol. Sci., Phys., B33, 209 (1994).

6. S. A. Gordeev, G. Y. Nikolaeva, and K. A. Prokhorov, Vysokomol. Soedin., Ser. A Ser. B, 38, 820 (1996).

7. S. S. Sheiko, M. Kunz, and M. Moller, Vysokomol. Soedin., Ser. A Ser. B, 35, 1903 (1993).

8. H. Y. Zhou and G. L. Wilkes, J. Mater. Sci., 33, 287 (1998).

9. B. Moginger, C. Lutz, A. Polsak, and U. Muller, Colloid Polym. Sci., 269, 535 (1991).

10. S. Onogi, T. Asada, and T. Takaki, J. Soc. Mater. Sci. Jpn. (in Japanese), 16, 746 (1967).

11. M. W. Mackenzie, "Advances Applied Fourier Transformation Infrared Spectroscopy”, John Wiley \& Sons, Inc., New York, N.Y., 1988.

12. G. Bayer, W. Hoffmann, and H. W. Siesler, Polymer, 21, 235 (1980).

13. Y. Shigematsu, A. Takada, N. Nemoto, and K. Nitta, Rev. Sci. Instrum., 72, 3927 (2001).

14. H. W. Siesler, Adv. Polym. Sci., 65, 1 (1984).

15. R. J. Samuels, Makromol. Chem. Suppl., 4, 241 (1981).

16. R. G. Snyder and J. H. Schachtschneider, Spectrochimica Acta, 20, 853 (1964).

17. H. W. Siesler and K. Holland-Moritz, "Infrared and Raman Spectroscopy of Polymers", Marcel Dekker, Inc., New York, N.Y., 1980.

18. S. Nomura, R. Ashitani, H. Matsuda, and L. Banda, Polymer, 42, 9045 (2001).

19. K. H. Nitta and M. J. Takayanagi, J. Polym. Sci., Part B: Polym. Phys., 38, 1037 (2000).

20. A. Peterlin, J. Mater. Sci., 6, 490 (1971).

21. R. C. Ball, M. Doi, S. F. Edward, and M. Warner, Polymer, 
22, 1010 (1981).

22. J. Sweeney, T. L. D. Collins, P. D. Coates, and R. A. Duckett, J. Appl. Polym. Sci., 72, 563 (1999).

23. J. Sweeney, T. L. D. Collins, P. D. Coates, and I. M. Ward, Polymer, 38, 5991 (1997).

24. J. Sweeney, T. L. D. Collins, P. D. Coates, A. P. Unwin, and I. M. Ward, ASME J. Eng. Mater. Tech., 119, 228 (1997).
25. V. Gaucher-Miri, P. Francois, and R. Seguela, J. Polym. Sci., Part B: Polym. Phys., 34, 1113 (1996).

26. A. R. Haynes and P. D. Coates, J. Mater. Sci., 31, 1843 (1996).

27. V. Gaucher-Miri and R. Seguela, Macromolecules, 30, 1158 (1997).

28. C. G'Sell, A. Dahoun, V. Favier, J. M. Hiver, M. J. Philippe, and G. R. Canova, Polym. Eng. Sci., 37, 1702 (1997). 